\title{
How I feed patients enterally
}

\section{By Simon P. Allison, University Hospital, Queen's Medical Centre, Clifton Boulevard, Nottingham $\mathrm{NG}_{7} 2 \mathrm{UH}$}

Before describing how I feed patients enterally, I would like to consider which patients should be fed in this way and what we are aiming to achieve. Providing the patient has a normal nutritional state before his illness, a few days' fasting makes little difference to the clinical outcome. So called 'clinical trials' which have purported to show otherwise do not bear close examination. Should patients, however, be in a malnourished state at the beginning of their illness, and have a condition which prevents adequate oral intake for more than a few days, there is an a priori reason for restoring nutritional intake since the laws of thermodynamics have yet to be repealed.

In honour of this meeting I have classified patients who need feeding according to Robert Burns. The first group come under the following heading: 'Some ha'e meat, but canna' eat'. Such patients have neurological or mechanical defects of swallowing, obstructive lesions of the upper gastrointestinal tract or surgery to the head and neck. Providing small bowel function is normal, enteral feeding by the nasogastric or jejunal route is cheap, safe and technically very satisfactory. Since these patients are usually unable to take anything by mouth, it is necessary to provide their total requirements via the tube.

The second group of patients come under the heading: 'Some ha'e meat and will na eat'. This is a group with varying degrees of anorexia whose oral intake is insufficient to satisfy their metabolic requirements. It includes patients with anorexia nervosa, the elderly post-operatively, and those with inflammatory-bowel disease. In health, most of us maintain a fairly close relation between food intake and weight, the link between the two being appetite. Some patients seem to reset this relation at a lower weight level which is often $15-20 \%$ below their usual body-weight. A typical case is the elderly patient who, 3 weeks post-operatively, remains apathetic, anorexic and losing weight. It is not sufficient to know that the patient is eating, we must know how much. For this reason we have devised simple charts with a column in which the nurse records the food which the patient actually eats and a column for the dietitian to sum the total nutrient intake. Frequently, we find that such patients are only eating $1600-2000 \mathrm{~kJ}$ (400 or 500 $\mathrm{kcal} / \mathrm{d}$ despite normal gastrointestinal function. Pressing such patients to eat more, causes distress and sours the relationship between them and the nursing staff. We therefore started to feed such patients by nasogastric tube while allowing them to eat whatever they liked. To our surprise, we found that the tube feeding caused restoration of appetite within 10 d (Bastow et al. 1985). Some patients, indeed, doubled their voluntary oral intake during this time, with rapid 
improvement in mood and gain in weight. In contrast, we found that parenteral nutrition could make patients feel better, but often resulted in anorexia so that it became difficult to wean patients straight from parenteral feeding to oral feeding. In some cases, it may be useful to bridge the gap with a few days of nasogastric feeding in order to restore appetite. There appears to be some effect of putting food into the stomach rather than into a vein which induces this appetite change. In this group of patients, we do not aim to supply the patient's total needs by nasogastric tube, but to supplement the oral intake in order to make up the deficit. As voluntary oral intake increases, so the nasogastric intake may be reduced and then withdrawn. For this procedure it is necessary to monitor the daily oral intake in the way that I have described.

The third group of patients are those with a short bowel, much of which has been resected because of disease. Often these patients have sufficient bowel to absorb energy and nitrogen, but have an inability to maintain salt and water balance due to the excessive losses even with small frequent meals.

\section{Equipment and practice}

The old style of nasogastric feeding involved the use of a Ryle's tube which was uncomfortable to pass, uncomfortable in position, and prone to cause oesophagitis. In spite of the fact that a plastic, fine-bore tube was described as early as $195^{2}$ by Fallis \& Barron (1952), it was not until recent years following the introduction by McMichael (1979) of the soft, plastic, fine-bore tube with its introducer wire, that the technique of tube feeding has become comfortable for the patient and free of complications (Allison et al. 1979). It used to be customary for nurses to give a small amount of food down the tube at intervals through the day. This proved demanding in nursing time, the feed was often forgotten, and nausea and diarrhoea were common. McMichael (1979) argued that if the feed were presented to the gut more slowly and gradually over a longer period, there would be less disturbance and osmotic effect, and hence less diarrhoea. This has been borne out in practice with continuous gravity feed from a reservoir via a drip-set, and more recently using a controlled infusion pump. In a study some years ago, Woolfson et al. (1976) found that this method saved a great deal of nursing time and reduced the incidence of nausea and diarrhoea. We also found, as did Silk (1983), that in the few cases where diarrhoea occurred, broad-spectrum antibiotics were to blame. There was some concern, using drip-feeds, that the nasogastric drip-set might accidently be connected to an intravenous cannula since the Luer fittings of the two were compatible. This was easily overcome by reversing the Luer fittings on tube-feeding sets so that the female Luer fitting is attached to the fine-bore tube and the male fitting to the giving set. The fine-bore tube with its introducer wire is easy to insert and can even be passed by the patients themselves, if nasogastric feeding should be necessary at home. To ensure correct positioning it is usually only necessary to squirt air down the tube with a syringe and listen over the epigastrium. If bubbles are heard then it is probably correctly positioned. However, 
in the unconscious or semi-conscious patient, I always perform an X-ray to make sure that the tube is in the stomach rather that the right main bronchus.

For both psychological and physical reasons, patients should not be tied to a drip-set all day, but should be mobile and free to do other things for most of the daylight hours. This is especially important in patients who are being fed at home and going to work during the day. From a purely metabolic point of view, immobility is a strong disadvantage. Schonheyder et al. (1954) performed the experiment of putting normal subjects to bed in plaster and showing the enormous negative $\mathrm{N}$ balance which resulted despite a substantial food intake. Muscles which are not active will waste whatever the feeding regimen, and the restoration of muscles, which have wasted during illness, will be greatly enhanced by activity combined with a large energy and $\mathrm{N}$ intake. It was with these thoughts in mind that we tried to confine tube feeding to a maximum of $12 \mathrm{~h}$ overnight. Giving between 2 and 3 litres of a feed containing $4.184 \mathrm{~kJ}(\mathrm{I} \mathrm{kcal}) / \mathrm{ml}$ results in disturbed nights from the diuresis induced by such a large volume. Accordingly, we tried to make the feed as concentrated as possible using glucose polymer and whole protein to give $8368 \mathrm{~kJ}$ ( $2000 \mathrm{kcal}$ ) in just over I litre of fluid with an osmolality of $400-420 \mathrm{mosmol} / \mathrm{kg}$. No diarrhoea was encountered even in patients with short-bowel syndrome. This was probably attributable to the use of a constant-infusion pump to maintain a slow and regular flow-rate over several hours. The enormous turnover of fluid in the gut ensures that the drops of feed are quickly diluted, so that no osmotic diarrhoea is produced, demonstrating the importance of osmol administered per unit time rather than osmol per unit volume. The giving of a single dose of $200 \mathrm{ml}$ of an isosmolar feed may well produce diarrhoea, whereas the slow administration of the same volume of hyperosmolar feed is free of such side effects. This is particularly important when we come to consider the management of short-bowel syndrome. I agree with Silk ( 1983 ) when he argues that gravity feeding is perfectly satisfactory for the majority of patients who are fed throughout the $24 \mathrm{~h}$, but I prefer to use a pump for the overnight feeding as it guarantees a steady rate of flow at a time when supervision is difficult and nursing time is at a premium. There has been debate about the appropriate size of reservoir to use for such feeds. A $500 \mathrm{ml}$ reservoir has been advocated on the grounds that, should any infection have been introduced even in warm surroundings, bacterial spoiling would not have time to occur. Being an advocate of overnight tube feeding, and starting with a sterile preparation anyway, I prefer a I. 5-2 litre reservoir which can contain the entire feed so that no disturbance is necessary during the night.

\section{Preparation and content of the feed}

We used to mix our feeds in the diet kitchen but became concerned about the introduction of pathogenic organisms at this stage. These may have caused problems in a few patients, but were certainly a potential source of serious cross-infection, particularly in the Intensive Care Unit. Bastow et al. (1982) showed that pathogenic bacteria, e.g. staphylococci and pseudomonas, introduced 
at this time could multiply to a concentration of over 1000000 organisms $/ \mathrm{ml}$ within a few hours. However, when a sterile proprietary feed was poured into the reservoir on the ward, there seemed to be no danger of infection and it was even possible to refill the reservoir at $12 \mathrm{~h}$ and detect no contamination at $24 \mathrm{~h}$. For this reason, as well as for its convenience, we now use the proprietary feeding mixtures of which there are several satisfactory examples on the market.

Proprietary mixtures mostly contain an energy concentration of 4.184 (I $\mathrm{kcal}) / \mathrm{ml}$ with an energy: $\mathrm{N}$ value somewhere between 150 and 200:1. For the overnight feeders, where a low volume and high concentration is desired, we mix an oligosaccharide concentrate such as Fortical with a high-protein preparation. This mixture is prepared by the nursing staff on the ward by pouring the separate sterile components directly into a sterile reservoir bag or bottle. The osmolality can be reduced by using glucose polymers instead of mono- or disaccharides and by using whole protein instead of amino acids. There was a great vogue at one time for so called elemental diets which contain amino acids rather than whole protein. As Silk ( 1983 ) has pointed out, this is a physiologically-unsound approach since $\mathrm{N}$ is absorbed by two pathways, not only in the form of individual amino acids but also as di- and tripeptides at the brush border of the small intestine. I can, therefore, see no case for the use of other than whole protein except in rare instances of severe pancreatic and bowel disease where a predigested preparation may well have an advantage: even then, a mixture of peptides and amino acids rather than amino acids on their own is preferable. Even in the short-bowel syndrome there is no advantage of elemental over polymeric diets (Andersson et al. 1984) and, indeed, some disadvantages, not least their excessive cost. A similar argument has prevailed over the ideal fat content of such feeds and the role of medium-chain triglycerides. Again it has been found in practice that these offer very little advantage except in a few cases of severe biliary, pancreatic or small-bowel disease.

I will not enumerate the vitamin and mineral requirements except to say that there are a variety of preparations with low or high sodium and potassium contents which are particularly suitable for different clinical situations. A low-Na preparation is needed for some patients with renal or heart failure, whereas a higher $\mathrm{Na}$ content may be desired in patients with high electrolyte losses from short-bowel syndrome or fistulas.

Returning to the 'Burns' classification'; I adopt a different policy for each group. In those with mechanical swallowing problems who are totally unable to take anything by mouth, I give a 2-3 litre isomolar feed over $16-24 \mathrm{~h}$, in order to give adequate fluid as well as food. In group 2 who may be able to take varying and, hopefully, increasing amounts of fluid and food by day, I feed them overnight with a I-I.5 litre hyperosmolar feed to supply $4184-8368 \mathrm{~kJ}$ (1000-2000 kcal). The patient is freed from the drip during the day to be mobile and to sit at a table and eat whatever he can manage. Since most such patients start in a nutritionally-depleted state, the high total energy and protein intake from the combination of tube feeding and oral intake ensures, in the mobile patient, a rapid 
restoration of function and of body tissue mass. In a recent controlled trial in elderly women suffering from a fractured femur, the feeding of a nutritionally-depleted group in this way resulted in a more rapid convalescence and recovery of idependent mobility, emphasizing the importance of body function rather than body mass as an index of clinical benefit from supplementary feeding (Bastow et al. 1983).

\section{Short-bowel syndrome}

Survival is possible following resection of the small bowel for trauma, mesenteric thrombosis or Crohn's disease with quite small residual lengths of bowel, e.g. $0.3-0.6 \mathrm{~m}$ of jejunum. Over several months such short lengths of bowel adapt by hypertrophy and magnification of their digestive and absorptive surfaces (Williamson, 1984). It may sometimes be necessary, therefore, to provide parenteral nutrition during this time and, in a few cases, permanent home parenteral nutrition. There are, however, patients in whom the amount of bowel remaining is sufficient to absorb enough energy and $\mathrm{N}$ from small, frequent meals and where the problem is more one of salt and mineral absorption. This is especially the case in those who have lost the ileum and large bowel and who have a short bowel combined with a jejunostomy stoma. Despite all attempts at dietary adjustment, such patients may have a persistent negative water, $\mathrm{Na}, \mathrm{K}$, calcium and magnesium balance which is incompatible with survival. They can often be managed very well by overnight nasogastric feeding at home which is certainly cheaper and simpler than the alternative of parenteral nutrition. I am currently treating a 45-year-old man who had most of his bowel resected for Crohn's disease and has $0.9-1.2 \mathrm{~m}$ of jejunum ending in a jejunostomy. Despite dietary adjustment, he continued in a negative balance of $\mathrm{I}$ litre small bowel juice/d and was $25 \%$ below his usual body-weight when we started to treat him I year ago. Since that time he has been giving himself overnight $1-1 \cdot 5$ litres of a fluid composed of $\mathrm{I}$ litre saline ( $9 \mathrm{~g}$ sodium chloride/l), $500 \mathrm{ml}$ water, two to three cans of Nutranel (a peptide-polysaccharide mixture) with the addition of $10 \mathrm{mmol}$ magnesium hydroxide. He measures his own weight daily to assess his fluid balance, and increases his overnight intake accordingly. This technique takes advantage of two physiological mechanisms. First, if salt and water are presented slowly enough to a short length of bowel, then absorption may be complete. Second, $\mathrm{Na}$ reabsorption is linked to, and enhanced by, glucose and amino acid absorption. This is the basis of all salt and sugar solutions used in the treatment of cholera and infant diarrhoea.

We were able to demonstrate this phenomenon more clearly in another patient who also had a short bowel with a jejunostomy but, following a number of surgical adventures, also had an isolated loop of jejunum and ileum which was $1.2 \mathrm{~m}$ long and opened at both ends onto the abdominal surface. The surgeon wished to leave the inflammation within the abdomen to subside before he attempted an anastomosis and we, therefore, accepted the patient for feeding for 1 year. For the first 3 months we fed her parenterally but, subsequently, we used an overnight 
perfusion of the isolated loop with a salt and glucose-polymer mixture. We were able to demonstate that with a slow infusion rate of $150 \mathrm{ml} / \mathrm{h}$, the inclusion of glucose polymer in the perfusion mixture enhanced $\mathrm{Na}$ and water reabsorption. With a little ingenuity, therefore, and some practical applications of physiology, the presence of a short bowel does not necessarily condemn the patient to home parenteral nutrition with all its attendant administrative difficulties.

\section{Home feeding}

I have already mentioned the ease with which patients and their relatives are able to pass a fine-bore tube and give themselves overnight tube feeding at home. Apart from the short-bowel patients, this has proved particularly useful in patients with neurological disease affecting swallowing and with mechanical obstruction in the mouth or upper gastrointestinal tract. It may make the difference between a comfortable existence and a miserable end associated with malnutrition and thirst. Many relatives feel helpless in the face of such severe disease and welcome the opportunity to play a part in the care of the patient, particularly when they can see that the results are so good.

\section{Monitoring of enteral feeding}

There is nothing magic about this. At one exteme with the home-fed patients, we just rely on weight and general well-being with a measurement of blood biochemistry and haematology every $2-3$ months or at any time the patient does not feel well. We often use anthropometric measurements of arm circumference and fat fold thickness to provide research information and initial nutritional assessment, but they are prone to large inter-observer error and a bed-side assessment by an experienced doctor or nurse may be just as good. In the Intensive Care Unit, or when large fluid losses occur from fistulas, then the daily measurement of blood biochemistry may be necessary. The best measure of water balance is change in weight. If the water balance is known, the $\mathrm{Na}$ balance may usually be inferred from the change in plasma $\mathrm{Na}$ concentration. With adequate hydration, serum $\mathrm{K}$ and $\mathrm{Mg}$ concentrations are a fair reflection of replacement requirements. Short-term changes in nutritional status may be reflected in the short, half-life plasma proteins such as thyroid-binding prealbumin, but for long-term follow-up, the serum albumin level is perfectly adequate. Both of these, of course, drop non-specifically following an operation or in the presence of acute illness due to distribution rather than metabolic factors. The recovery of protein levels to normal is, however, affected by nutritional state.

\section{Complications of tube feeding}

If the technique is carried out properly, complications should be virtually non-existent. Nausea and diarrhoea virtually disappear with slow administration, although diarrhoea may be a problem in the presence of broad-spectrum antibiotics: the administration of codein phosphate will counteract this. We have not found reflux with aspiration pneumonia to be a major problem, but it is always 
a worry in recumbent patients with clouded consciousness. An incompetent cardia causes reflux and a diminished cough reflex allows aspiration of feed into the lungs.

\section{REFERENCES}

Allison, S. P., Walford, S., Todorovic, V. \& Elliott, E. T. (1979). Research and Clinical Forums I, 49-57.

Andersson, H., Bosaeus, I., Ellegard, L., Hallgren, B., Hulten, L. \& Magnusson, O. (1984). Clinical Nutrition 3, 183-189.

Bastow, M. D., Greaves, P. \& Allison, S. P. (1982). Human Nutrition: Applied Nutrition 36A, 213-217.

Bastow, M. D., Rawling8, J. \& Allison, S. P. (1983). British Medical Fournal 287, 1589-1 592.

Bastow, M. D., Rawlings, J. \& Allison, S. P. (1985). Clinical Nutrition 4, 7-1 2.

Fallis, L. S. \& Barron, J. (1952). Archives of Surgery 65, 373 .

McMichael, H. B. (1979). Research and Clinical Forums 1, 25-28.

Schonheyder, F., Heilskov, N. S. C. \& Olesen, K. (1954). Scandinavian fournal of Clinical and Laboratory Investigations $6,178$.

Silk, D. B. A. (1983). In Surgical Nutrition, p. I9 [J. E. Fischer, editor]. Boston and Toronto: Little, Brown \& Co.

Williamson, R. C. N. (1984). Clinical Nutrition 3, 169.

Woolfson, A. M. J., Ricketts, C. R., Hardy, S. M., Saour, J. M., Pollard, B. J. \& Allison, S. P. (1976). Postgraduate Medical Journal 52, 678-682. 